\title{
Oral submucous fibrosis recuperated with prosthodontic approach- a case report
}

\author{
Dr.Shital Jalandar Sonune ${ }^{1}$, Dr. Shankar P. Dange ${ }^{2}$ \\ ${ }^{I}$ (Dept. of Prosthodontics, Dr D.Y.Patil Dental college, Pimpri, Pune, M.S., India) \\ ${ }^{2}$ (Dept. of Prosthodontics, Govt. Dental College, Aurangabad, M.S., India)
}

\begin{abstract}
Oral submucous fibrosis is a disease due to chronic, insidious change in fibroelasticity, characterized by burning sensation in the oral cavity, blanching and stiffening of the oral mucosa \& oropharynx leading to trismus \& inability to open the mouth. It is predominately seen in Indians \& other Asians. The pathogenesis of the disease is thought to be multifactorial. A wide range of treatment consisting of drug management, surgical therapy \& physiotherapy have been attempted till date with varying degree of benefit, but none of them have proved to be a cure for this disease. In literatures very less information is given about prosthetic treatment of patients with oral submucous fibrosis.

In this case report, an edentulous patient with oral submucous fibrosis is presented \& a prosthodontic rehabilitation technique that improves retention, stability, and esthetics \& comfort aspect with a conservative approach is described.
\end{abstract}

Key words: Conservative management, Oral submucous fibrosis, Prosthodontic rehabilitation

\section{Introduction:}

Oral submucous fibrosis is defined as a chronic disease of oral mucosa characterized by inflammation and progressive fibrosis of the lamina propria and deeper connective tissue layers. The pathogenesis of the disease is not well established, but is believed to be multifactorial. ${ }^{1}$ A number of factors trigger the disease process by causing juxtaepithelial inflammatory reaction in the oral mucosa. Suggested contributory factors include areca nut chewing, ingestion of chillies, nutritional deficiencies, genetic $\&$ immunologic processes and other factors. Oral submucous fibrosis is a potential premalignant condition with an incidence of oral cancer in $3-7.6 \%$ cases. $^{2}$ The presenting symptoms of Oral submucous fibrosis are burning pain, progressive inability to open the mouth, difficulty in mastication and swallowing.

It is most common between 20 to 40 years of age with a female to male ratio of $3: 1{ }^{3}$ When it affects geriatric patients with partial or complete edentulism; the task of restoring function becomes challenge to the Prosthodontist because of the patient's clinical presentation. This clinical case report describes the treatment modality for a patient of Oral submucous fibrosis who needed rehabilitation with complete denture.

\section{Outline Of Case:}

A 56 year old completely edentulous female patient reported to the Department of Prosthetic Dentistry with the chief complaint of restricted mouth opening, inability to masticate \& burning sensation of mouth. The patient gave history of pan chewing 3-4 times a day since last 15 years but had discontinued this habit over a period of time. Extra oral examination showed the patient had a restricted mouth of about $25 \mathrm{~mm}$ (figure 1).

Intraorally, the patient presented with edentulous maxillary and mandibular ridges with fibrous band (figure 2) extending from buccal mucosa to ridge resulting in shallow buccal vestibule. The buccal \& labial mucosa was found to be pale and showed evidence of palpable thick fibrous bands. Also insufficient depth of lingual vestibule was seen with atrophic mucosa and salivary gland hypo-function.

A clinical diagnosis of Oral submucous fibrosis with complete edentulism was made. As the patient had already discontinued the habit of pan chewing so the patient was advised physiotherapy (opening and closing the mouth wide with maximum efforts for 15-20 minutes atleast 4 times a day). After the necessary preliminary management, the definitive prosthetic treatment was started in a conservative manner. The patient was made to use a saliva substitute (Wetmouth, ICPA, India) before making impressions. Small size stock trays were selected and primary impressions made with irreversible hydrocolloid (Tropicalgin, Zhermack, Italy). The special tray was fabricated with borders overall $2 \mathrm{~mm}$ short of vestibule.

The minimal impression technique was used for making final impression so no border molding was done. Functional border molding using green stick compound was carried out only with posterior palatal seal area and alveolingual sulcus, were mucosa was resilient. Due to the patient's complaint of burning sensation in the mouth, medium body elastomeric impression material (Impregum ${ }^{\mathrm{TM}}$ Soft Impression Material, 3M ESPE, India)was preferred for making wash impression (figure $2 \& 3$ ). 
The next clinical step constituted recording and transferring maxillomandibular relation. The maxillary \& mandibular occlusal rims were adjusted and the jaw relation was recorded \& secured on an articulator. As the fibrous bands in the buccal vestibule were very thick and exerted pressure on the lateral borders of the ridge it was decided to reduce occlusal forces on the ridge hence artificial denture teeth (Acry-Rock, Ruthenium) of small mold size were selected and arranged accordingly. To facilitate arrangement of posterior teeth, second molars on either arch \& side were deleted from the teeth arrangement (figure 4). During try-in the esthetic, phonetics, vertical dimension and occlusion were checked. The denture was processed in heat cure acrylic resin in the usual manner (figure 5), dentures were placed in patient's mouth and occlusion \& extensions were checked. Follow-up visits were scheduled for 24 hrs., one week, 3 months \& six months later, for any minor adjustment. During follow-up visits the patient was found to be amended with the dentures (figure 6) as she was advised to use denture adhesives while eating her meals.

\section{Discussion:}

Oral submucous fibrosis is a pre-cancerous condition occurring chiefly in south east asia. ${ }^{4}$ It was first reported in a group of East Indian women by Schwartz ${ }^{1}$, but has been extensively studied by Pindborg ${ }^{5}$ and his associates. Oral submucous fibrosis is defined as an insidious chronic disease affected any part of the oral cavity and sometimes the pharynx, although occasionally preceded by and/or associated with vesicle formation. It is always associated with juxtaepithelial inflammatory reaction followed by fibroelastic changes of lamina propria with epithelial atrophy causing progressive restriction of oral opening.

The etiology of oral submucous fibrosis is obscure. Some evidences suggest that it may be related to betel nut chewing, vitamin B deficiency or peculiar dietary component viz. chillies and spices commonly used in India. ${ }^{6,7}$ The disease is characterized by burning sensation of mouth accompanied by formation of vesicles \& ulceration. Xerostomia is evident in these patient's. The mucosa becomes blanched and opaque, fibrotic bands appear usually involving buccal mucosa, soft palate, lips and tongue causing restricted mouth opening.

The different treatment modalities for oral submucous fibrosis are vitamin and protein supplements, microwave diathermy, local injections, oral corticosone, surgical excision, skin graft and lateral tongue flap. ${ }^{8}$ There is currently no definitive treatment for this condition and with increased general life expectancy, there is a higher percentage of individuals reaching old age with Oral submucous fibrosis at different grades of intensity. Inspite of the high prevalence of this condition in geriatric patients in our country, there is a lacuna in literature regarding prosthetic management of completely edentulous patients suffering from Oral submucous fibrosis.

In the present case conventional complete denture prosthesis was fabricated. The patient presented with dense fibrous bands within the oral cavity extending from buccal mucosa to the ridge hence a routine sectional border molding was not done, except for the posterior palatal seal area and alveolingual sulcus were the fibrous bands are resilient. As the fibrous bands are elastic in nature a wash impression is recorded with open mouth minimal pressure technique. Minimal pressure impression believes in the principle of recording the tissues with minimum pressure $\&$ the borders are kept short of the peripheries. No border molding is done. The retention is by adhesion, cohesion $\&$ interfacial surface tension. ${ }^{9}$

Jaw relation was secured in usual manner. Artificial teeth function as a unit on their base. The masticatory forces in complete denture patients are distributed on the entire denture bearing area through resilient mucosa. In this case as the underlying mucosa is not resilient it affects the number \& position of teeth. Hence to reduce the masticatory forces \& facilitate space for posterior teeth second molars were eliminated. A need based treatment approach was considered and the patient was treated conservatively.

\section{Conclusion:}

In literatures a lot can be found about reviews, researches \& various treatment modalities for oral submucous fibrosis, but very less citations are present about prosthodontic rehabilitation, impression material \& impression technique selection for edentulous patients affected with oral submucous fibrosis. In the present case report, taking into consideration the signs, symptoms and needs of the patient suffering from oral submucous fibrosis, a conventional complete denture was fabricated with minimal pressure technique using polyether elastomeric impression material.

\section{References:}

[1] Canniff JP, Harvey W, Harris M. Oral submucous fibrosis: its pathogenesis and management. Br Dent J 1986;160:429-34

[2] Rajendran R. Oral submucous fibrosis: etiology, pathogenesis and future research. Bulletin of the World Health Organization 1994;72(6):985-96.

[3] Jayanthi V, Probert CS, Sher KS, Mayberry JF. Oral submucous fibrosis: a preventable disease. Gut 1992;33:4-6.

[4] Simpson W. Submucous fibrosis. Br J of Oral Surg 1969;6:96.

[5] Pindborg JJ, Sirsat SM. Oral submucous fibrosis. Oral Surg 1966;22:764-79.

[6] Rajendra R, Vijay kumar R, Vasudan OM. An alternate pathogenic pathway of oral submucous fibrosis. Medical Hypothesis 1989;30:35-37. 
[7] Wahi EN. The epidemiology of oral and prophyryngeal cancer. Bulletin of the World Health Organization 1968;38:495-521.

[8] Tabassum S, Nagda SJ. Oral submucous fibrosis-case report of a prosthodontic rehabilitation. J Indian Prostho Society 2001;1:35-37.

[9] Ram SM. Prosthodontics at a glance: questions \& answers. ${ }^{\text {st }}$ edn.

Figure 1. Restricted mouth opening

\section{LIST OF LEGENDS:}

Figure 2. Edentulous ridges with non-resilient mucosa

Figure 3. Final impression in polyether impression material (mandibular)

Figure 4. Final impression in polyether impression material (maxillary)

Figure 5. Occlusal surfaces of finished dentures

Figure 6. Patients smile with prosthesis 